\title{
The Cylindrical Envelope Projection Model Applied to SE Images of Curved Surfaces and Comparison with AFM Evaluations
}

\author{
Massimo Antognozzi and Ugo Valdrè \\ Centre for Electron Microscopy, Department of Physics and INFM-CNR, via Irnerio 46, \\ 40126 Bologna, Italy
}

(Received March 23; accepted April 12, 1995)

\begin{abstract}
The Cylindrical Envelope Projection Model (CEPM) has been extended to images of curved objects (e.g. spheres), obtained in a SEM by means of SEs, in order to improve the accuracy of measurements (down to a few \%). The test objects have been calibrated by means of an AFM equipped with home-made nanotips. A simple rule for measuring the diameter of spheres and cylinders from SE $y$-modulated traces is given. The rule is applicable to specimens of medium $(Z \geq 25)$ and high atomic number.
\end{abstract}

\section{Introduction}

The various imaging signals used in a scanning electron microscope [such as those produced by secondary electrons (SEs) and backscattered electrons] are the result of a complex interaction of the primary electron beam with the specimen which involves, in particular, the geometry of the sample. Hence the resulting images are usually only qualitatively and intuitively interpretable, and it is not always correct to directly derive quantitative information from them. This is true, in particular, with the measurement of distances between 3D-details when edge effects are of paramount importance, as in the case of high gradient topographic features, linear and curved steps.

In fact, the interaction between the electron beam and the specimen details under measurement is still present when the beam axis is beyond the outer surface of the details. In addition, the position of the signal intensity peak is not an indication of the specimen edge. The error associated with these measurements is related to the geometry of the SE generation volume, and has a value of the order of the electron beam diameter, even if use is made of $y$-modulation profiles. In the case of features of size less than $1 \mu \mathrm{m}$ and a beam diameter of $50 \mathrm{~nm}$, the error is not less than $10 \%$.

Accurate measurements are essential in precision metrology and in each case where the beam diameter is a substantial fraction (depending on the required accuracy) of the distance to be measured. This applies to both the cases of free surfaces and of interfaces emerging at the specimen surface. 
It is therefore important to develop a practical and reliable method to simulate the beam/specimen interaction at the sample edges, to be used for accurate measurements of sizes and distances. This problem has been tackled by several people [1-3] by making some simplifying assumptions. The proposed methods, however, fail to provide accurate answers in the case of steep steps. Recently, an improved method, the cylindrical envelope projection model (CEPM) has been introduced in order to estimate the size of micro-steps in Si from SE images for metrology purposes [4].

The present work expands and applies the use of the CEP model to cases of greater geometrical complexities, not previously examined. The signal considered is that of the SEs, the most widely used signal in scanning electron microscopy.

\section{The Basis of the Cylindrical Envelope Projection (CEP) Model}

The major factors affecting the intensities and the dimensions of SE image details are: the primary electron beam energy, the angle between the beam axis and the specimen surface normal at the point of incidence, the specimen shape and nature and, to a lesser extent, the orientation of the specimen plane with respect to the E-T detector. They may be divided into geometrical and physical factors, which are interconnected by the diffusion volume. The latter may be found by Monte Carlo simulations [2] or through the modified diffusion model [3]. However, the former requires large computation time and the latter does not give sufficient weight to the sample geometry when steep slants are present, and leads to discrepancies between the true and measured values [4].

The CEPM has proved to be effective in the metrology of inclined surfaces [4]. The geometrical and physical factors are brought about by considering a cylinder around the beam axis (of suitable radius, which depends on the electron range, the depth of complete diffusion and the beam radius) within which the SEs are produced in amount which is supposed to be proportional to the area of intersection between the cylinder and the specimen surface.

The cylinder radius, $R_{\mathrm{c}}$, is the radius of the circular intersection of the diffusion ellipsoid with a plane, parallel to the $(x, y)$-plane, which is perpendicular to the axis of rotation of the ellipsoid ( $z$-axis) (Fig. 1). $R_{\mathrm{c}}$ is given by [4]:

$$
R_{\mathrm{c}}(\mu \mathrm{m})=(R+r) \cdot\left(R^{2}-Z_{\mathrm{d}}^{2}\right)^{1 / 2} / R
$$

where $r(\mu \mathrm{m})$ is the beam radius, $R(\mu \mathrm{m})=Z_{\mathrm{r}}-Z_{\mathrm{d}}$ the radius of the diffusion volume and

$$
Z_{\mathrm{d}}(\mu \mathrm{m})=40 Z_{\mathrm{r}} /(7 Z)
$$

is the penetration depth [5]. $Z$ is the specimen atomic number and $Z_{\mathrm{r}}$ the primary electron range given by Young [6]:

$$
Z_{\mathrm{r}}(\mu \mathrm{m})=\left[0.115\left(\mu \mathrm{m} \mathrm{cm}^{-3} \mathrm{~g}^{-1} \mathrm{keV}^{-1}\right) E^{1.35}\right] / \rho
$$

$\rho\left(\mathrm{g} \mathrm{cm}^{-3}\right)$ is the specimen specific gravity and $E(\mathrm{keV})$ the incident electron energy.

Although the CEP model has been extended to several geometries (cylinders, cones and conical etch pits, and spheres), the work reported refers to: (i), the measurement of diameters of small spheres, the most complex case, and (ii), the presentation of a simple rule of accurate diameter measurements via SE $y$-modulated line traces, without recourse to computer modelling.

The SE emitting surface is given, in the CEP model, by the area, $S$, of the figure of intersection of the sampling cylinder and the surface of the sphere (Fig. 2) for each value of the horizontal 


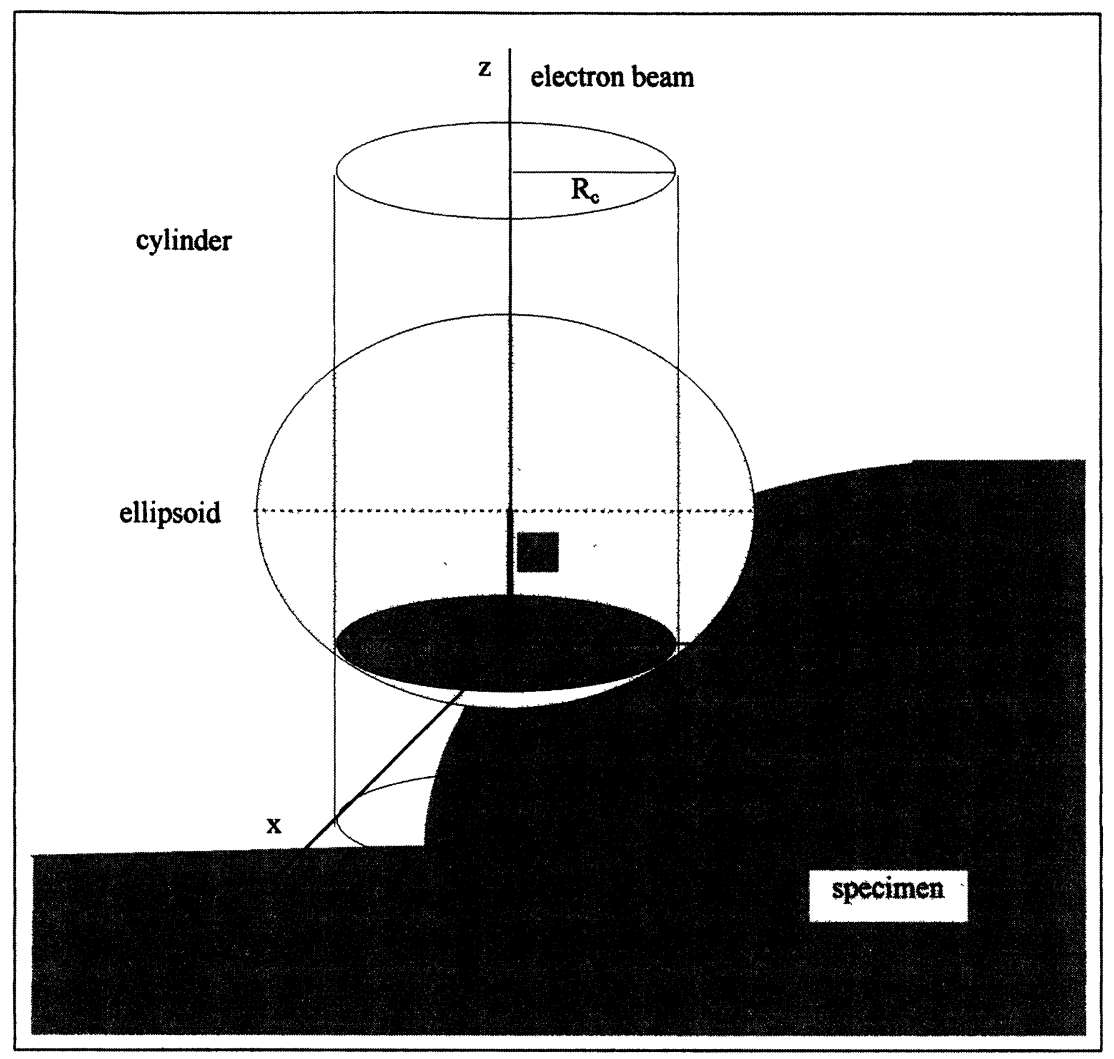

Fig. 1. - Showing the principle of the CEP model and some of the relevant quantities.

coordinate. To be noted that in the case previously treated [4] this intersection is constant along the slant. In general, $S$ is given by a surface integral which involves the $x$ - and $y$-coordinates, and variable boundary conditions for each scanning line. In the case of the determination of the diameter of a sphere, it suffices, however, to calculate the theoretical line profile across a diametral plane.

All the results obtained from SE images in a SEM have been compared with simulated images (a computer program has been written for use in a PC), with data provided by the suppliers of the spheres, when available, and with measurements performed in an AFM utilizing home-made super-tips. In this case, the accuracy of the measurements is better by a factor of 10 with respect to that obtainable with ordinary pyramidal tips [as can be seen by comparing Figs. $3 \mathrm{a}$ and $3 \mathrm{~b}$ ].

\section{Preparation, Calibration of the Specimens and Results}

3.1 SPECIMEN PREPARATION - In order to check the accuracy of the CEPM for spheres, several types of specimens were prepared, measured in an AFM and used as standard.

They are latex spheres from dilute suspensions, belonging to different batches and comprising a range of diameters (from $200 \mathrm{~nm}$ to $1 \mu \mathrm{m}$ ). The spheres were either deposited on graphite flakes or mica sheets, then placed on an AFM disc-shaped specimen holder and coated with a layer of $\mathrm{Au}(15 \div 30 \mathrm{~nm}$ thick), to avoid charging up in the SEM. Coating was performed both by sputtering 


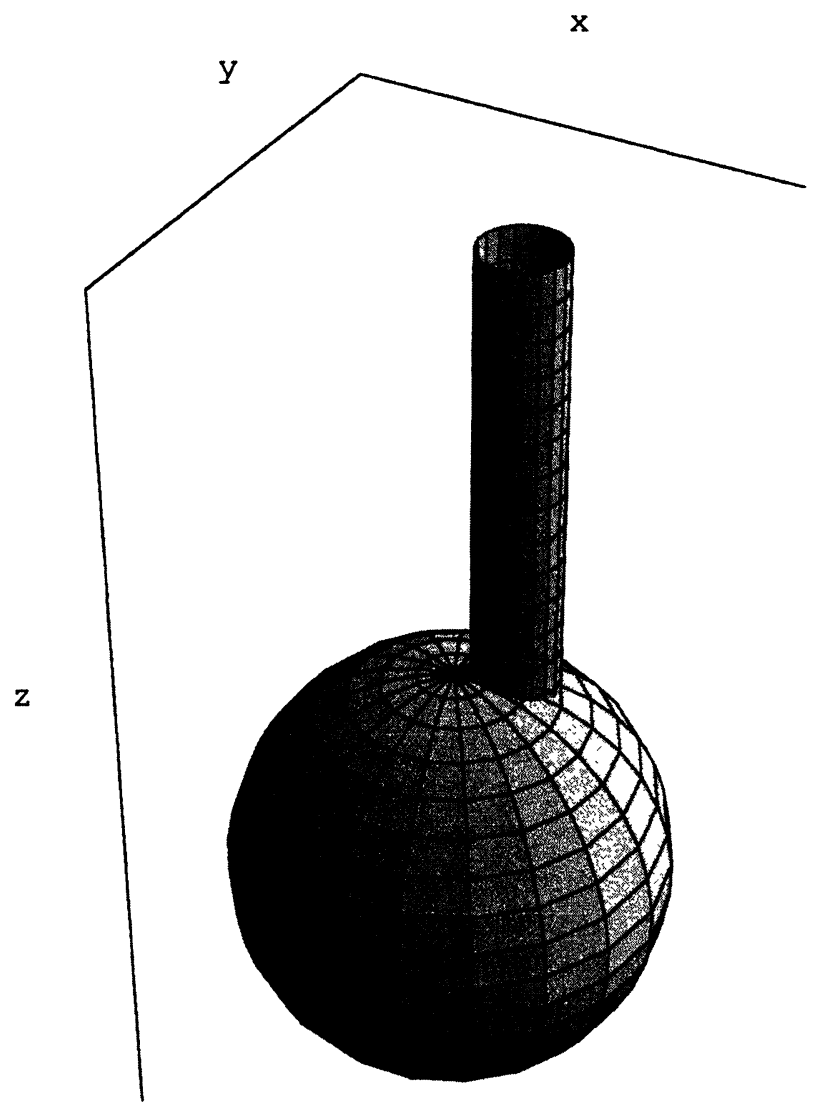

Fig. 2. - Schematic drawing of the CEP model applied to a sphere. The average surface slope increases on moving from the sphere top towards right. Consequently, the emission area increases until it drops rapidly to zero as the electron beam leaves the ball edge.

and by evaporation of gold. Evaporation with a rotating specimen and a coating angle of about $45^{\circ}$ was preferred, a posteriori, to sputtering because of the smaller granularity of the films and consistency of results (Figs. 4a, b, c). In the case of mica, the substrate was scratched to produce a cross-grating of a few lines in order to be able to identify specific spheres during the observation in both SEM and AFM. The mica was coated with a metal film to avoid the dragging of the spheres during scanning in the AFM.

Some latex spheres were mounted on grids for calibration in a transmission electron microscope (TEM). Since difficulties were experienced in the transfer and observation of the specimens in both SEM and AFM, the use of a TEM was discontinued.

3.2 Calibration of Specimens Used as Size Standards - Since the diameter of the latex spheres is specified by the manufacturer as a mean value of the batch, there may be changes of dimension from sphere to sphere. In addition, the spheres must be metal coated to prevent charging-up effects during SEM observations; this in turn affects the ball size. In order to perform accurate SEM measurements, the diameter of single spheres had to be measured with high precision in a direct and independent way. To this purpose the AFM was used since it is capable 

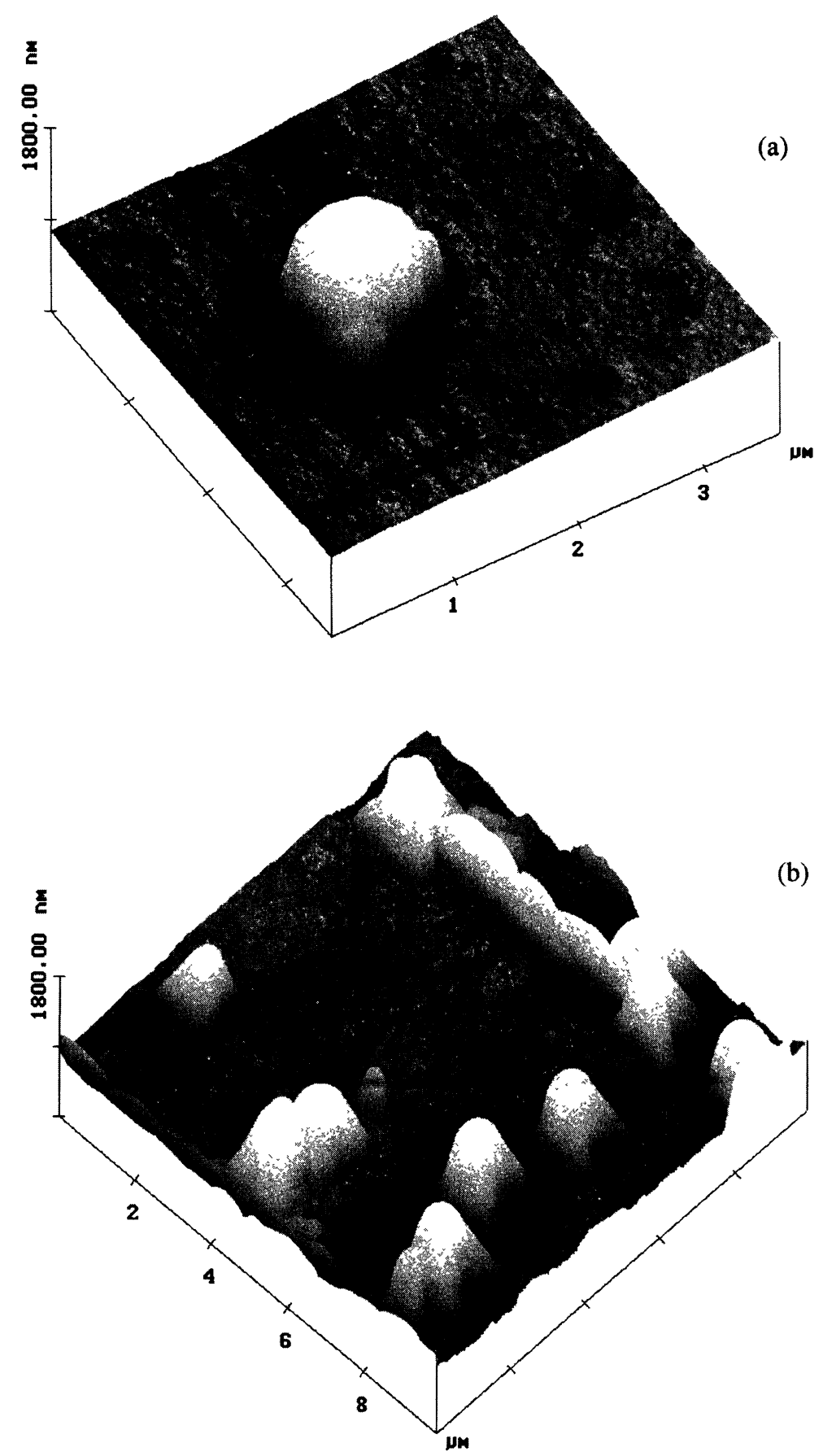

Fig. 3. - a) AFM image of a latex sphere obtained with a carbon nano-tip of $10 \mathrm{~nm}$ radius. Surface details of the sphere have been used as identification markers (see also Fig. 5 and Fig. 7b). b) AFM image of spheres obtained with a standard pyramidal tip. It is clear that the instrument has convoluted the spheres with the tip shape. 


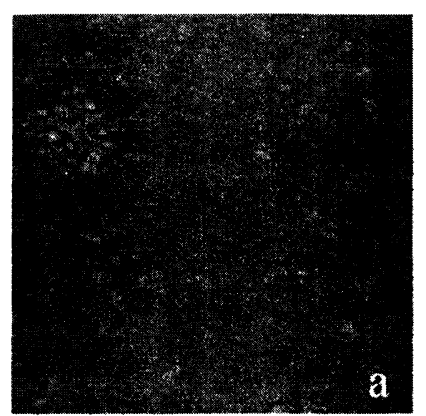

$2 \mu \mathrm{m}$

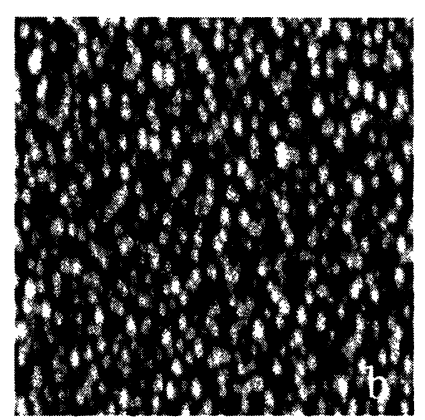

$2 \mu \mathrm{m}$

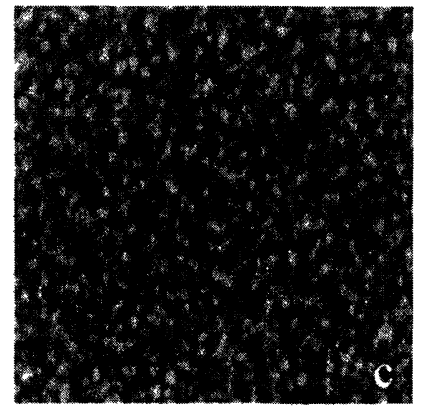

$2 \mu \mathrm{m}$

Fig. 4. - a) Low resolution AFM image of an evaporated Au film. b), c) Au films obtained under (allegedly) identical sputtering conditions, observed by low resolution AFM under the same imaging conditions, to show the unconsistency of the results.

of high resolution and since the specimen could be directly transferred from it to the SEM with the possibility of observing the same area.

Two problems had then to be faced: (i) to accurately measure the diameter of given spheres; (ii) to recognize specific balls in performing observation in the SEM, AFM and vice versa. The latter problem has been solved by the set of lines engraved in the mica substrate, by selecting specific pattern in the arrangement of the sphere and finally by choosing spheres well recognizable by means of specific details (Figs. 3a, 5 and $7 b$ ).

ลับ-

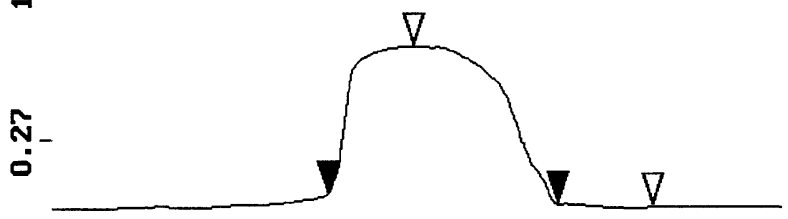

م⿱⺈

Horiz distance (L) $1.183 \mathrm{\mu m}$

Vert distance

$955.67 \mathrm{~nm}$

3.00

\section{NM}

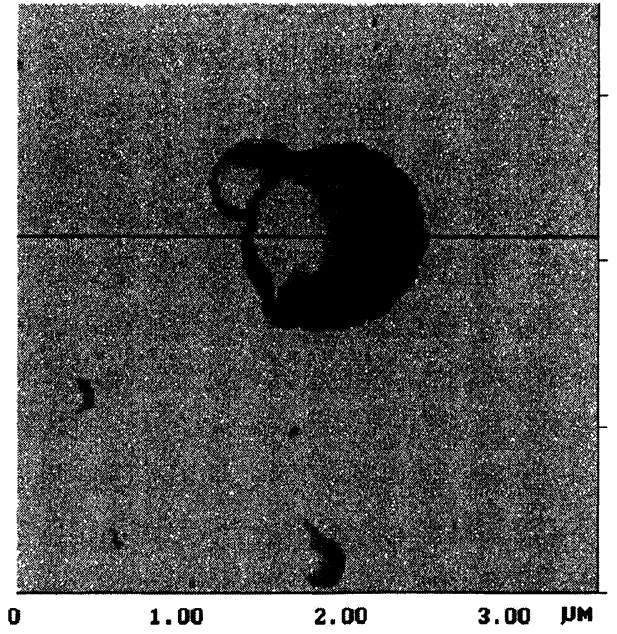

1.00

2.00

$3.00 \mathrm{NM}$

Fig. 5. - (left). Diametral line profile of the latex sphere imaged on the right in an AFM equipped with a nano-tip. The triangular markers indicate the horizontal distance $L$ (dark markers) and the vertical coordinate (bright markers); the latter can be used as an alternative method to derive the sphere diameter. 


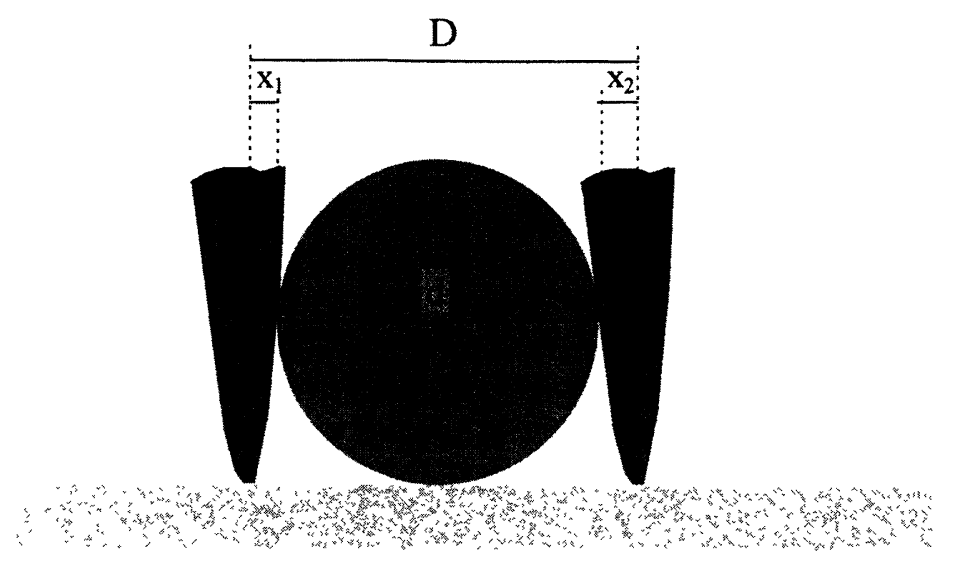

Fig. 6. - Model used to work out the diameter $d=D-\left(x_{1}+x_{2}\right)$ of a sphere, with correction for the tip size. The tip has been assumed to be asymmetrical $\left(x_{1} \neq x_{2}\right)$; its size was measured in a TEM.

Once the selected spheres had been traced, the measurement of their diameter brings problem (i). As an example, let us consider Figure 5 which shows an AFM image of an Au coated latex sphere (right) and a line trace (profile, to the left) in a plane containing a sphere diametral plane. The measure of this diameter must take into account the probe size and shape (Fig. 6). In the instance is $d=D-\left(x_{1}+x_{2}\right)=992 \pm 7 \mathrm{~nm}$. This value is taken as the right diameter for the calculations of the theoretical profile, to be compared with the experimental SE profile. The above values have been found consistent with the diameters, $d_{\mathrm{v}}$, derived by measuring the height of the AFM profiles and adding to it twice the thickness of the Au coating $(2 \cdot 15 \pm 2 \mathrm{~nm})$. In the reported example is $d_{\mathrm{v}}=985 \pm 3 \mathrm{~nm}$. The small difference between the two values is attributable to a slight compression of the ball due to the coating.

3.3 RESULTS - Figure 7a (continuous line) shows the calculated profile for the reported values of the parameters. This profile is compared with the experimental $y$-modulated line scan (dashed line) taken in the SEM for the same latex sphere (Fig. 7b, reported in the graph of Fig. 7a to ease the comparison). As can be seen, the agreement is rather good on the right hand side.

The discrepancy on the left hand side is due to the presence of a "blob" which was used (together with others) to identify the latex sphere. The experimental profile is higher than the calculated one in the region between the peaks; it is attributed to the granularity of the ball surface.

The results show that within the range of accelerating voltages 4 to $30 \mathrm{kV}$, neither the peak-topeak distance, nor the base diameter $b$ (Fig. 7a), the width at half peak height and the distance derived by the thumb rule based on the intersections of the tangents to the profile at the flex points give the value of the sphere diameter. The last three values overestimate the actual size, whereas the peak-to-peak distance underestimates it. It is also found that, for values of $Z$ above 25 , a better estimate of the diameter is given by the distance $h$ between the points of intersection of the experimental $y$-modulated fine traces and the horizontal line drawn through the mid-point between the maximum and the minimum of the profile (e.g., Figs. 7, 8).

\section{Conclusions}

The validity of the Cylindrical Envelope Projection Model (CEPM) has been tested in a scanning electron microscope (SEM) by using the secondary electron (SE) signal. The specimens used were 


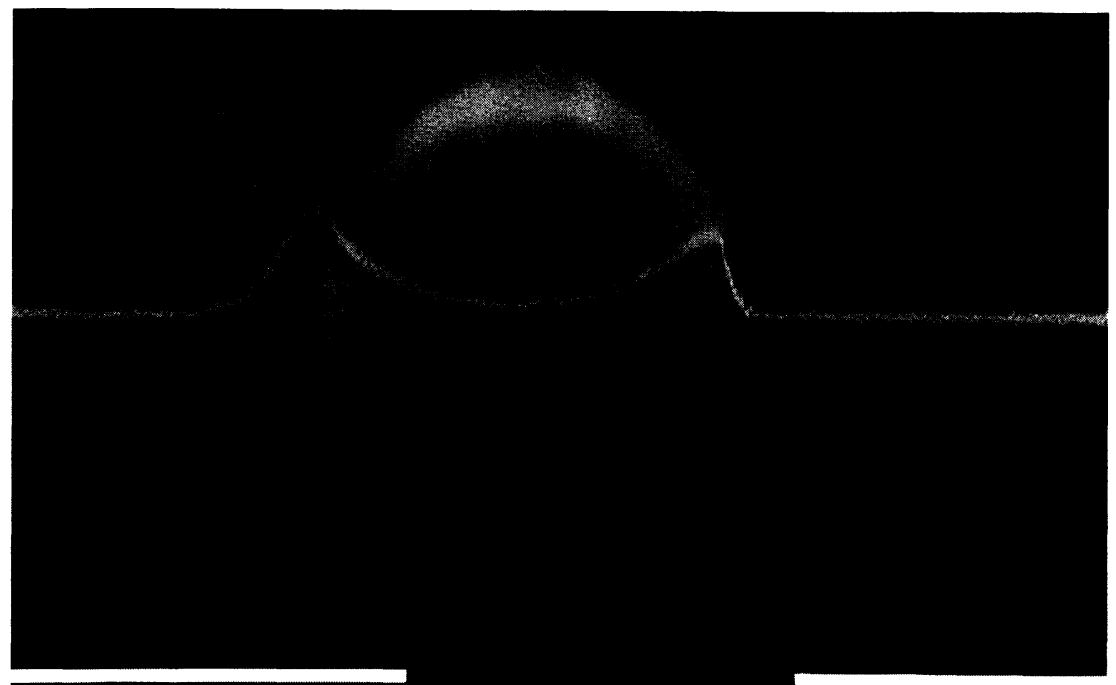

\section{$1 \mathrm{\mu m} 4.0 \mathrm{kV} \quad 4.00 \mathrm{~m} 40015 / 002$ NM50}

a)

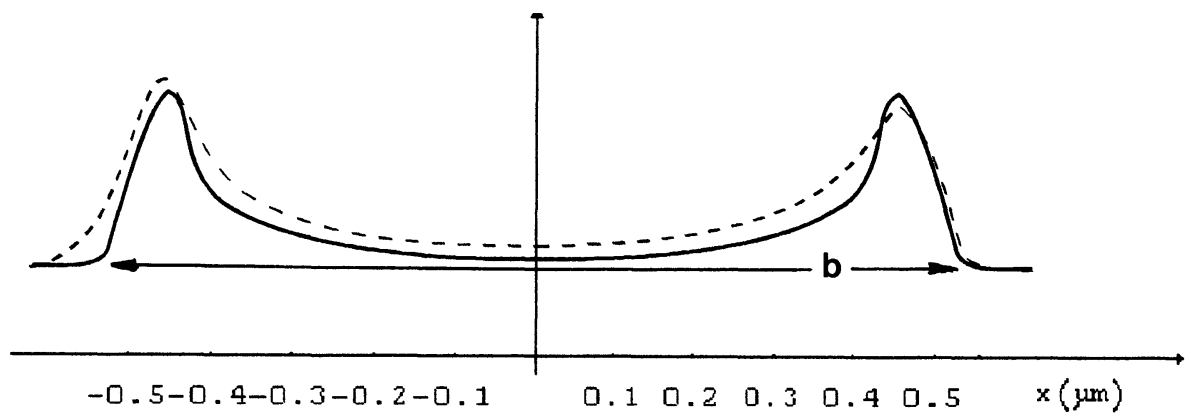

- - experimental line profile

- theorical line profile

b)

Fig. 7. - a) Calculated profile (continuous line) and experimental $y$-modulated line trace (dashed line) for a sphere of effective diameter of $992 \pm 7 \mathrm{~nm}$. The experimental parameters are: accelerating voltage, $4 \mathrm{kV}$; beam diameter, $50 \mathrm{~nm} ; R_{\mathrm{c}}=60 \mathrm{~nm}$. b) SE image of the latex sphere, with superimposed a diametral, $y$-modulated line trace, as for (a).

latex spheres whose diameter had been accurately measured (about 1\% accuracy) in an Atomic Force Microscope (AFM) equipped with carbon nano-tips. By using reference marks and specific specimen details, it has been possible to exactly identify the same spheres in both the AFM and SEM. 


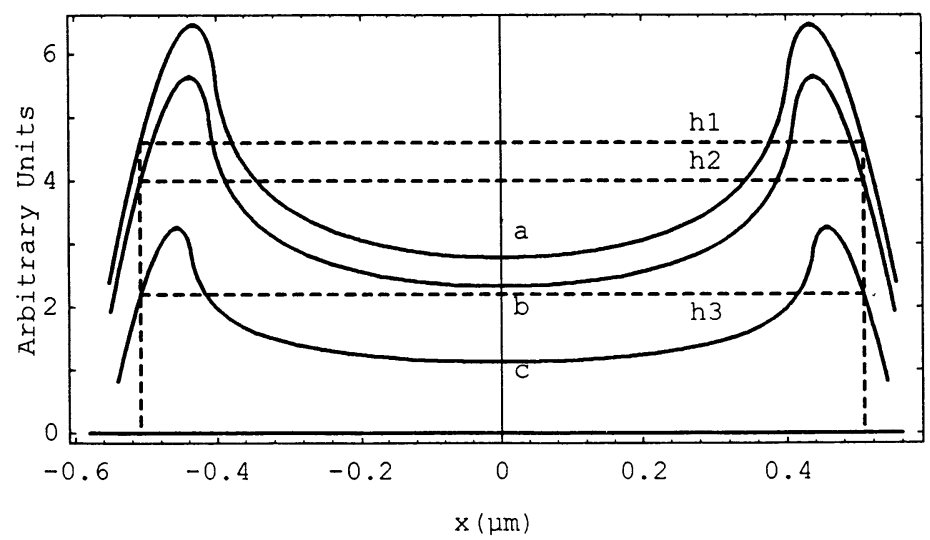

Fig. 8. -- CEPM calculated profiles of spheres of different materials having the same diameter (992 $\mathrm{nm}$ ). $\mathrm{a}=\mathrm{Fe} ; \mathrm{b}=\mathrm{Ag} ; \mathrm{c}=\mathrm{Au}$. It happens that horizontal lines $\mathrm{h} 1, \mathrm{~h} 2$ and $\mathrm{h} 3$, drawn through points that are placed at half the vertical separation between the peaks and the minima of curves $a, b$ and $c$ respectively, meet the outer parts of these curves at points having the same abscissa. This value $(1016 \mathrm{~nm})$ is taken as the radius of the sphere.

By comparing the theoretical profiles derived by the application of the CEPM method to spheres of known size to experimental $y$-modulated line traces across a diametral plane, the following empirical rule and results have been obtained for SEM accelerating voltages ranging from 4 to $30 \mathrm{kV}$.

(i) An accurate value of the diameter of spheres (and of cylinders) can be derived from the experimental $y$-modulated line trace across a diametral plane by taking the distance $h$ at half depth (i.e. the width at half height, when the latter is measured from the "true" background).

(ii) The accuracy is about $4 \%$.

(iii) The results are strictly applicable to specimens of high and medium atomic number $Z$ (down to about $Z=25$ ), although it has been found that rule (i) provides the correct answer for $\mathrm{Al}$ and $\mathrm{Si}$, too.

(iv) The various empirical rules commonly used for size measurements (such as peak-to-peak, meeting points of the tangents to the flexes, width at half peak) are less accurate; the former underestimates the size by about $15 \%$, whereas the others overestimate the actual value by 10 to $20 \%$.

Several obvious applications of the results obtained may be foreseen, such as measurements of fibres, tubules, globules, cavities, vesicles, bubbles, indents and etch pits. We were particularly interested in the problem of the measurements of the radius of curvature of nano-emitters for field emission guns and of the radius of super-tips for atomic force microscopy.

\section{Acknowledgements}

This work has been partly supported by MURST, Rome, and by the European Union. 


\section{References}

[1] Postek M.T. and Joy D.C., J. Res. NBS 92 (1987) 205.

[2] Joy D.C., J. Microsc. 147 (1987) 51.

[3] Kuni A., Kembo Y., Yoshiyama K. and Aoki N., Proc. Microelectronics Measur. Tech. Seminar (1980) p. 67.

[4] Hatsuzawa T., J. Meas. Sci. Technol. 4 (1993) 842.

[5] Archard G.D., J. Appl. Phys. 32 (1961) 8.

[6] Young J.R., J. Appl. Phys. 28 (1957) 5. 